\title{
Behaviour of free falling viscoelastic liquid jets.
}

\author{
Christophe Tirel, Marie-Charlotte Renoult*, Christophe Dumouchel, Jean-Bernard Blaisot \\ Normandie Université, Université et INSA de Rouen, CNRS - CORIA \\ 76801 Saint-Etienne du Rouvray, France \\ *Corresponding author: renoultm@coria.fr
}

\begin{abstract}
In a recent work, a protocol to measure the relaxation time of dilute polymer solutions, known to be challenging, has been established [1]. This protocol is based on a 2D multi-scale description of free-falling low velocity viscoelastic liquid jets. Although the relaxation time reached an asymptotic value for high jet velocities, a significant dependence with the jet velocity is observed for low velocities. The present work reconsiders these previous experimental data using a 3D multi-scale analysis in order to identify the origin of the dependence between the relaxation time and the jet velocity. The 3D analysis demonstrates the importance of a velocity-dependent coalescence mechanism in the jet behaviour. Thanks to a simple model of jet deformation it is demonstrated that this coalescence mechanism prevents the elasto-capillary contraction of the smallest scales from occurring when the jet velocity is reduced.
\end{abstract}

\section{Keywords}

Dilute polymer solutions, Viscoelasticity, Jet break-up, Multi-scale analysis

\section{Introduction}

Contrary to Newtonian fluids, the atomisation of a cylindrical jet of a viscoelastic solution presents long life time cylindrical ligaments connecting spherical beads, the so called beads-on-a-string (BOAS) structures [2]. These structures are observed at times prior jet breakup. It is well known that the thinning evolution of the ligaments diameter is controlled by the elasticity of the liquid [3]. The decrease is exponential, with a relaxation time depending only on the physical properties of the liquid. The measurement of this relaxation time is also known to be particularly challenging for dilute polymer solutions [4] for which the relaxation time is very short (micro-second or under). Previous studies [5-8] have investigated the possibility to use a cylindrical jet to measure this quantity, a method that could be suitable to probe very short times [4]. Yet, it was shown that the relaxation time obtained with this method depends on the jet operating conditions, i.e. the jet velocity, the amplitude and the frequency of the initial disturbance [6-8], thus limiting the development of this experimental method. Until recently, new results [4] suggest that an operating domain where these dependences vanish may exist.

In this context, a multi-scale analysis of the behaviour of free falling jets of a dilute viscoelastic solution was performed, allowing measurements of the relaxation time [1]. The dynamics of the smaller scales was monitored and an exponential decrease was observed from which a relaxation time was extracted. It was found that the measured relaxation time decreases with increasing jet velocity, reaching a plateau for sufficiently high velocities. This behaviour was related to the influence of the initial amplitude disturbance as reported in [6-8]. The higher jet velocity was imposed, the larger initial amplitude was observed on jet images and the lower relaxation time was measured in agreement with [6-8]. To date, the dependence of the relaxation time with the jet velocity (or the initial amplitude disturbance) remains unexplained. The objective of the present paper is thus to complete this previous work [1] by performing a 3D multi-scale analysis for these free falling jets of dilute viscoelastic solutions in order to gain a deeper insight into the jet velocity dependence. A model of jet deformation is used in complement to enrich the $3 \mathrm{D}$ analysis. Section 2 summarizes the previous investigation and describes the 3D multi-scale analysis and the model. Section 3 presents the results and the application of the model. The article ends with a conclusion.

\section{Jet images: acquisition and analysis}

This section summarizes the experimental work and the 2D multi-scale analysis that are presented in detail in [1]. It also introduces the 3D multi-scale analysis and the model of jet deformation which constitute the new aspect of the present study.

The experimental setup produces free falling jets of a low-viscosity viscoelastic polymer solution containing $5 \mathrm{ppm}$ of Poly(ethylene oxide) (PEO) dissolved into a solvent (density $\rho=989 \mathrm{~kg} / \mathrm{m}^{3}$; shear viscosity $\mu=1.34 \mathrm{mPa}$.s; surface tension $\sigma=48.9 \mathrm{mN} / \mathrm{m}$ ). The liquid was kept in a pressurized tank and fed an injector with a cylindrical nozzle whose diameter is $D_{o r}=200 \mu \mathrm{m}$. Images of the free falling jets were obtained with a high spatial resolution camera and a light source of short duration (20 ns) used in a diffused backlight illumination optical arrangement. 
The field of view covered by the images was $6.56 \mathrm{~mm} \times 5.54 \mathrm{~mm}$ and the spatial resolution was equal to $2.56 \mu \mathrm{m} /$ pixel. A 3D displacement system was used to translate the injector to be able to visualize the jets on their full length, i.e., from the nozzle exit down to the breakup point.

The parameter of the experiment was the injection pressure that ranged from 0.05 to 1 bar. The mean jet velocity $V_{q}$ was obtained by dividing the measured volume flow rate by the jet section area $\pi D_{j}^{2} / 4$ where the mean jet diameter $D_{j}$ was measured on the jet within the three first millimeters after the nozzle exit. The mean jet velocity ranged from $1.9 \mathrm{~m} / \mathrm{s}$ to $10 \mathrm{~m} / \mathrm{s}$. For each injection pressure and for each position of the injector, 204 images decorrelated in time were recorded and the subsequent analysis was statistical. A reconstructed full jet is shown in Fig. 1. The jet issuing from the nozzle is slightly deformed. At around $16 \mathrm{~mm}$ a sinusoidal disturbance appears. This disturbance grows and rearranges to the expected beads-on-a-string pattern (BOAS) which remains visible until breakup occurs.

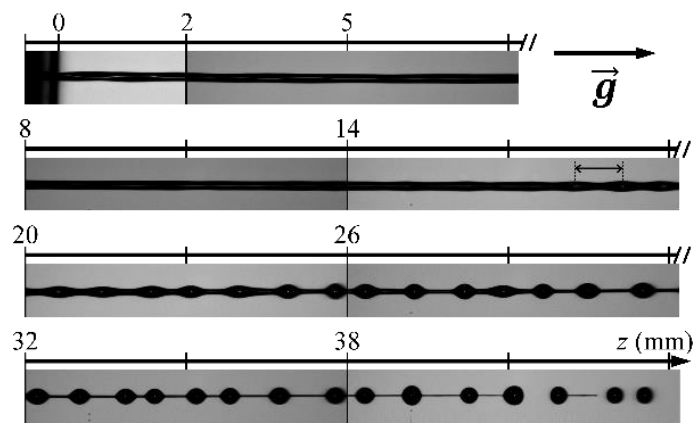

Figure 1. Reconstructed jet (Fluid: polymer solution, Mean jet velocity $V_{q}=8.58 \mathrm{~m} / \mathrm{s}$ )

The analysis of these images used a 2D multi-scale description of the jet. This description consisted in measuring the surface-based cumulative scale function $E_{2}(d, z)$ as a function of the position $z$ (see [1]). First, image treatments were applied to provide two-level images where the liquid appeared as black pixels on a white background. Second, a rectangular analyzing window delimiting a portion of the jet was positioned at a distance $z$ from the nozzle exit. Third, the surface-based cumulative scale function $E_{2}(d, z)$ of the jet portion delimited by the analyzing window was measured. Introduced in several previous works (see [9-11] for instance), the measurement of this function is inspired from the Euclidean Distance Mapping method to determine fractal dimension [12] and consists in applying successive erosion operations at a scale $d$ ranging from 0 to infinity. The erosion operation consists in removing a strip of width $d / 2$ around the whole system. The cumulative scale function $E_{2}(d, z)$ is the ratio of surface area of the removed strip on the total surface area of the local jet portion. For the scale $d=0$, the surface area removed by erosion is equal to zero and $E_{2}(0, z)=0$. For a sufficiently large scale, the surface area removed by erosion is equal to the total surface area of the local jet portion and $E_{2}(d, z)=1$. Between these two scales, $E_{2}(d, z)$ monotonously increases. A more detailed definition of the cumulative scale function is available in [9-11]. At each $z$ position, the measurement was performed on 204 images and an average cumulative function was calculated. The spatial evolution of the average cumulative scale function was obtained by sliding the analyzing window from the top of the jet (just under the nozzle) down to the bottom (in the spray region). The size of the analyzing window was chosen of the order of the length of the BOAS pattern i.e. 390 pixels for every velocity. Furthermore, 390 pixels appeared also appropriate for the shift of the analyzing window along the $z$ axis. The spatial series of local cumulative scale functions in space was transformed as a temporal series using the equivalent time $t$ defined as:

$$
t=\frac{\sqrt{V_{q}^{2}+2 g z}-V_{q}}{g}
$$

where $g$ is the gravitational acceleration. When $V_{q}^{2}>>2 g z$ (Froude number $>>1$ ), Eq. (1) simplifies as $t=z / V_{q}$. The 2D multi-scale analysis concentrated on the 2D scale-distribution $e_{2}(d, t)$ which is the first derivative of the cumulative function in the scale space: $e_{2}(d, t)=d E_{2}(d, t) / d d$. This distribution is equal to the ratio of the perimeter of the eroded local system at scale $d$ on twice the total surface area of the local system. The temporal evolution of the scale distribution $e_{2}(d, t)$ gives information on the physical mechanisms involved in the shape evolution of the jet. To illustrate this, one can consider the temporal derivative of the scale distribution as shown for instance in Fig. 2 $\left(V_{q}=1.9 \mathrm{~m} / \mathrm{s}, t=6.3 \mathrm{~ms}\right.$, BOAS pattern). This derivative is equal to zero for three characteristic scales noted $d_{1}, d_{2}$ and $d_{3}$. The $d_{3}$ scale is the maximum scale $d_{\max }$ of the system. The scale $d_{4}$ for which the derivative is a minimum is a characteristic scale. It has been demonstrated ([11] for instance) that the specific behavior of the derivative in the small scale range $\left(d<d_{1}\right)$ in Fig. 2 denotes the presence of a thinning cylindrical ligament. The scale $d_{1}$ appears 
as a characteristic scale of the dynamics of the ligament. The temporal evolution of $d_{1}$ for the free viscoelastic jets reported a decrease according to three regimes [1]. The second one is an exponential regime which corresponds to an elastic-controlled capillary thinning behavior [1], i.e.:

$$
d_{1} \propto \exp \left(-\frac{t}{3 t_{r}}\right)
$$

where $t_{r}$ is the relaxation time of the viscoelastic solution. The relaxation time was measured from Eq. (2) as a function of the mean jet velocity $V_{q}[7]$. Figure 3 reports the results by plotting the Deborah number $D_{e}=t_{r} / t_{\sigma}$ as a function of the square root of the jet Weber number We where:

$$
t_{\sigma}=\sqrt{\frac{\rho D_{j}^{3}}{8 \sigma}} \quad W e=\frac{\rho V_{q}^{2} D_{j}}{\sigma}
$$

Figure 3 shows a dependence between $t_{r}$ and the mean jet velocity. Nevertheless, we note that an asymptotic value is reached when We increases. This value is $t_{r}=0.44 \mathrm{~ms}$ and is believed to be the relaxation time of the solution. In order to obtain a better understanding of the behaviour of low-velocity free-falling viscoelastic jets, we suggest completing the previous analysis by a 3D multi-scale approach. This is the purpose of the present work.

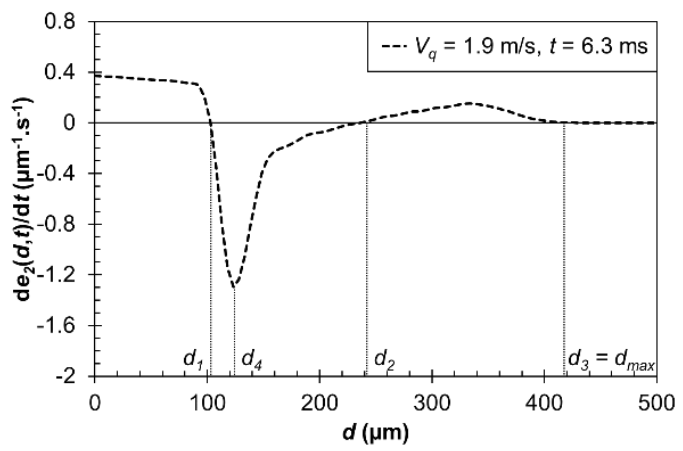

Figure 2. Temporal derivative of the scale distribution. Introduction of characteristic scales

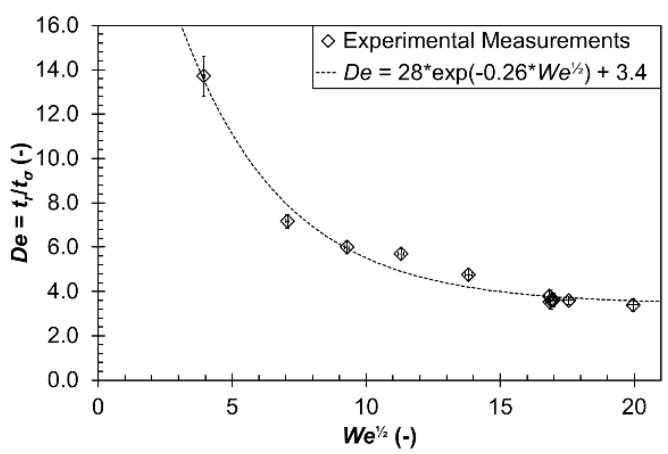

Figure 3. Deborah number $D_{e}$ as a function of the Weber number (from [7])

In the case where 3D system information is available, the 3D generalization of the 2D multi-scale description summarized above is straightforward (see $[11,13]$ for applications). The system is characterized by its volume and the erosion operation removes the peripheral volume of a $d / 2$ thickness from the system boundary. The cumulative distribution $E_{3}(d, t)$ is the ratio of the volume removed by the erosion at scale $d$ on the total volume of the system. The scale distribution $e_{3}(d, t)$ defined as the first derivative of the cumulative distribution $E_{3}(d, t)$ in the scale space is equal to the ratio of the surface area of the eroded system at scale $d$ on twice the total volume of the system. Therefore, $e_{3}(0, t)$ is directly proportional to the specific-surface-area (surface of the interface per unit volume) introduced by Evers [14].

In the present work, the studied free liquid jets are assumed axisymmetric. This assumption seems reasonable since the capillary instability is known as an axisymmetric mechanism. For each jet portion delimited by the analyzing window, this hypothesis allows to calculate the jet volume and surface by integration for each successive values of the scale $d$. Then, the 3D multi-scale description uses these two pieces of information to calculate the scale distribution $e_{3}(d, t)$. After the analysis of all the images, we determine $V(t)$ which is the local average volume of the jet portion delimited by the analyzing window, and $e_{3}(d, t)$ which is the local average volume-based scale distribution.

One of the main objectives of the 3D multi-scale approach is to describe the behavior of the jet when it is shaped as a BOAS pattern. In this case, the jet can be described as the composition of two simple systems: a cylinder of diameter $D_{1}(t)$ and volume $V_{1}(t)$ (system 1 ) and a sphere of diameter $D_{2}(t)$ (volume $V_{2}(t)$, system 2). The volumebased scale distributions for these two systems are:

$$
\text { System } 1 \quad e_{3}(d, t)=\frac{2}{D_{1}(t)}\left(1-\frac{d}{D_{1}(t)}\right) \quad \text { for } d<D_{1}(t)
$$


System $2 \quad e_{3}(d, t)=\frac{3}{D_{2}(t)}\left(1-\frac{d}{D_{2}(t)}\right)^{2} \quad$ for $d<D_{2}(t)$

The similarity between these two expressions must be noted. Furthermore, the scale distribution of a cylinder shows a first order dependence with the scale and a sphere shows a second order one. This observation suggests that a system whose shape is between a cylinder and a sphere would report a scale dependence order between 1 and 2 . Therefore, since the free falling jet evolves from a cylindrical system to an arrangement of a cylinder and a sphere (see Fig. 1), its scale distribution can be modelled as:

$$
\begin{aligned}
& e_{3}(d, t)=\alpha(t) \frac{2}{D_{1}(t)}\left(1-\frac{d}{D_{1}(t)}\right)+(1-\alpha(t)) \frac{n}{D_{2}(t)}\left(1-\frac{d}{D_{2}(t)}\right)^{n-1} \quad \text { for } d<D_{1}(t) \\
& e_{3}(d, t)=(1-\alpha(t)) \frac{n}{D_{2}(t)}\left(1-\frac{d}{D_{2}(t)}\right)^{n-1} \quad \text { for } D_{1}(t)<d<D_{2}(t)
\end{aligned}
$$

In Eq.(6), the parameter $n$ varies from 2 to 3 as time increases. At early times the jet is cylindrical and $n=2$. Then, when a part of the jet swells up, $n$ increases. It then approaches 3 when the swollen part is almost a sphere. In Eq.(6), the parameter $\alpha(t)$ represents the relative volume of the ligament part of the jet: $\alpha(t)=V_{1}(t) /\left(V_{1}(t)+V_{2}(t)\right)$. The knowledge of this parameter may provide a better characterization of the behaviour of the ligaments in the BOAS pattern. In particular, if the evolution of the BOAS is due to a capillary contraction of the string controlled by the viscoelasticity of the solution, the volume $V_{1}(t)+V_{2}(t)$ should be constant, the diameter of the string should decrease as Eq. (2) and therefore, the dynamic of the parameter $\alpha(t)$ is expected to be:

$$
\alpha(t) \propto \exp \left(-\frac{2 t}{3 t_{r}}\right)
$$

Therefore the model expressed by Eq. (6) can bring precious information about the analysis of the 3D multi-scale description of the free falling viscoelastic liquid jets.

\section{Results}

Figure 4 presents the temporal evolution of $V(t)$, the average volume of liquid delimited by the analysing window, for three jet velocities. $\left(V(t)\right.$ is divided by $V\left(t_{i}\right)$ where the initial time ti corresponds to the top position of the analysing window.) The time is made dimensionless by using the breakup time $t_{B \cup}$ whose average value has been determined for every jet velocity. We see that $V(t)$ is rather constant during time for each velocity. This shows that 204 images are enough to perform statistical analysis. Note however that for $V_{q}=4.5 \mathrm{~m} / \mathrm{s}$ and $9.0 \mathrm{~m} / \mathrm{s}, V(t)$ slightly decreases first before becoming constant. This evolution results from the fact that the assumption of axisymmetry is not fully satisfied at the beginning for these jets that are initially subjected to a slight deformation. This remains insignificant for the subsequent analysis.

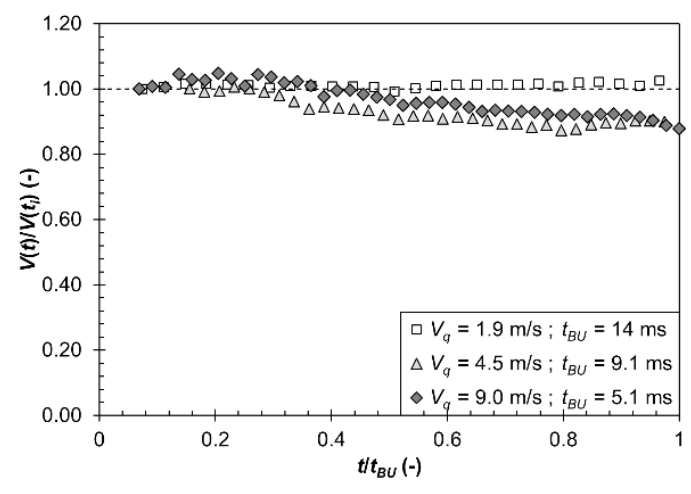

Figure 4. Temporal evolution of the ratio $V(t) / V\left(t_{i}\right)$ as a function of the mean jet velocity $V_{q}\left(t_{B U}\right.$ is the breakup time)

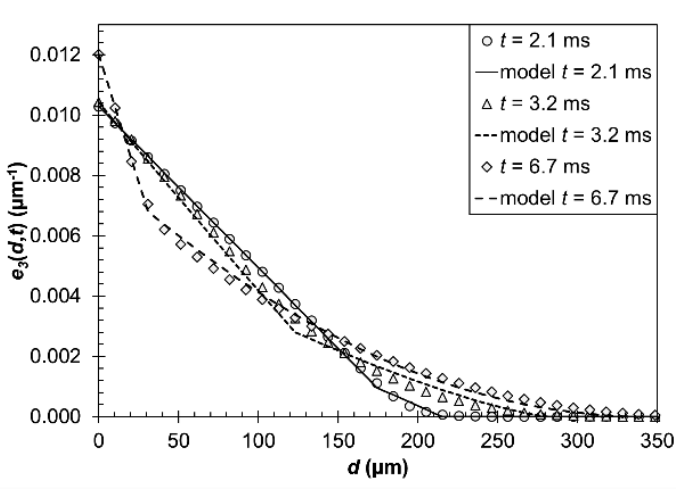

Figure 5. Volume-based scale distribution $e_{3}(d, t)$ as a function of time $\left(V_{q}=5.7 \mathrm{~m} / \mathrm{s}, t_{B U}=7.2 \mathrm{~ms}\right.$, dots: measurements; lines: model)

Figure 5 shows an example of the temporal evolution of the volume-based scale distribution $e_{3}(d, t)\left(V_{q}=5.7 \mathrm{~m} / \mathrm{s}\right)$. At $t=2.1 \mathrm{~ms}$, the scale distribution is linear which, according to Eq. (4), signifies that the jet is still a cylinder. As times goes, the scale distribution loses this linearity indicating that the jet experiences a deformation. In the large scale region, we note a continuous increase of the maximum scale $d_{\max }$. This illustrates the presence of a thickening 
mechanism that is due to the growth of swells along the jet. In the small scale region, the distribution remains linear with the scale and the absolute value of the slope increases with time. These two behaviours reveal the presence of a thinning cylindrical ligament.

As mentioned in the previous section, $e_{3}(0, t)$ is equivalent to the specific-surface-area of the system, i.e., the interface area per unit volume. The temporal evolution of this quantity appears to be very much dependent on the mean jet velocity. This is illustrated in Fig. 6 -a where the product $e_{3}(0, t) D_{j} / 2$ is plotted as a function of the ratio $t / t_{B U}$ for several mean jet velocities. At early times, since the jets are cylindrical for every velocity, $e_{3}(0, t) D_{j} / 2$ should be equal to 1 (see Eq. (4)). Figure 6-a reports this value for all cases. As time increases, two types of evolution are identified. For the high velocities $(5.7 \mathrm{~m} / \mathrm{s}$ to $9 \mathrm{~m} / \mathrm{s}$ in Fig. $6-\mathrm{a}), e_{3}(0, t) D_{j} / 2$ increases up to a local maximum at around $t / t_{B U}=0.5$ and then decreases before increasing again when the breakup is approached. The first increase corresponds to the deformation of the cylindrical jet caused by the growth of the swells (as for the Newtonian case [11]) and the subsequent decrease occurs during the evolution of the BOAS pattern. This indicates that the evolution of the BOAS is an interface reduction mechanism. The last increase before the breakup is moderate for $7.0 \mathrm{~m} / \mathrm{s}$ and $9.0 \mathrm{~m} / \mathrm{s}$ but is much pronounced for $5.7 \mathrm{~m} / \mathrm{s}$.

For the other velocities, the evolution of $e_{3}(0, t) D_{j} / 2$ is different: it shows a continuous increase until the breakup is reached. Furthermore, the variations of $e_{3}(0, t) D_{j} / 2$ are much higher than for the highest velocities. Such an increase of the specific-surface-area suggests that the system is very much deformed, i.e., it is far less spherical.

This is verified by considering the product $S P=e_{3}(0, t) d_{\max }(t) / 2$. $S P$ is a shape parameter. It is equal to 1 for a cylinder (Eq. 4) and to 1.5 for a sphere (Eq. 5). For a system composed of a cylinder of diameter $D_{1}(t)$ plus a sphere of diameter $d_{\max }(t)$ (with $d_{\max }>D_{1}(t)$ ), the parameter $S P$ is equal to (see Eq. (6)):

$$
S P=\alpha(t) \frac{d_{\max }(t)}{D_{1}(t)}+\frac{3}{2}(1-\alpha(t))
$$

Figure 6-b shows the temporal evolution of the shape parameter $S P$ for the same velocities as in Fig. 6-a. The horizontal dash lines indicate $S P$ for a cylinder and for a sphere. For all velocities, $S P=1$ at the initial time. Then, the increase of $S P$ until the breakup is approached $\left(t / t_{B U}=1\right)$ depends on the mean jet velocity. For the high velocities $(5.7 \mathrm{~m} / \mathrm{s}$ to $9 \mathrm{~m} / \mathrm{s}$ in Fig. 6-b), we note an inflection of $S P$ when the value 1.5 is exceeded. Then, $S P$ moderately increases until the breakup is reached. (Once again, we note that the jet with $V_{q}=5.7 \mathrm{~m} / \mathrm{s}$ shows a different behaviour near the breakup with a more pronounced increase than for the two other velocities.) The fact that $S P$ remains close to 1.5 at breakup means that the surface-area of the system is mainly brought by the beads that are close to spheres. In other words, the amount of surface-area brought by the strings is almost negligible. For the low velocities $(1.9 \mathrm{~m} / \mathrm{s}$ to $4.5 \mathrm{~m} / \mathrm{s}$ in Fig. 6-b), the inflection after $S P=1.5$ is far less pronounced than for higher velocities and the subsequent increase of $S P$ is far more pronounced. Equation (8) indicates that high values of $S P$ are due to a combination of a high $d_{\max } / D_{1}$ ratio and a non-negligible parameter $\alpha(t)$. Therefore, contrary to the previous case, the surface-area of the system at breakup is not brought by spherical beads only and the contribution of the string is not negligible anymore.

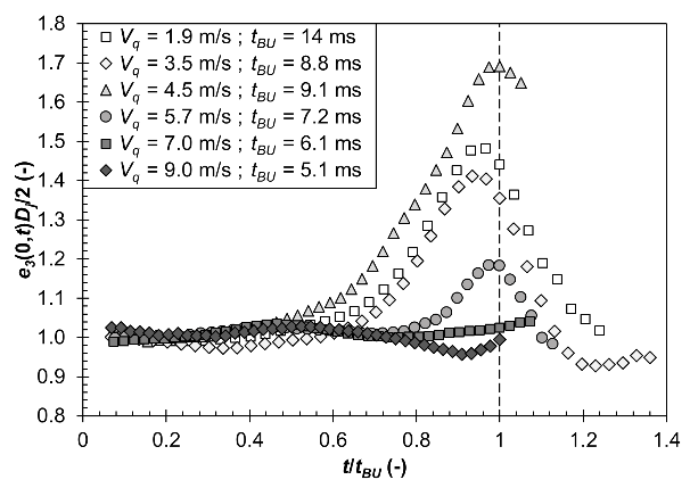

Figure 6-a. Temporal evolution of the specific-surface-area. Influence of the mean jet velocity

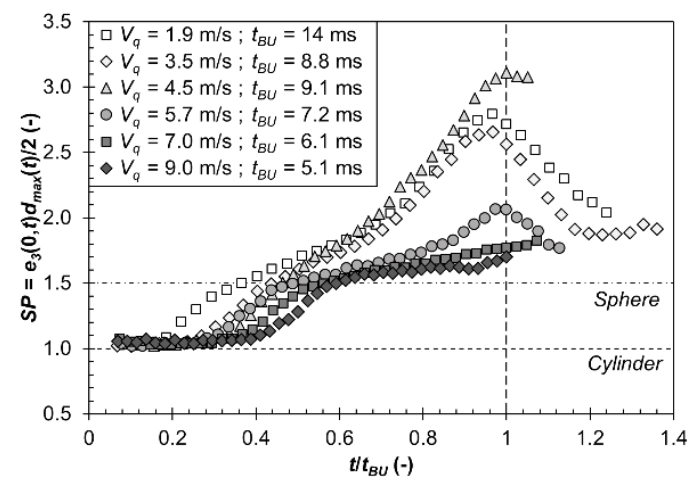

Figure 6-b. Temporal evolution of the shape parameter $S P$. Influence of the mean jet velocity

As a complement of the results shown in Fig. 6-a and 6-b, Fig. 7 presents the pdf (probability density function) of the maximum scale $d_{\max }$ of the drops visible after the breakup. We clearly see in this figure that the pdf of $d_{\text {max }}$ depends on the mean jet velocity: it is shifted to larger $d_{\max }$ as the velocity decreases. According to Eq. (8) this could contribute to the high values of $S P$. Furthermore, since at low velocity the beads are larger than at high velocity, they could be also more deformed and could explain the high specific-surface-area reported in Fig. 6. 
A close examination of the images revealed the presence of a mechanism of coalescence of consecutive swells along the jet. This mechanism is visible for each velocity. It is illustrated in Fig. $8\left(V_{q}=1.9 \mathrm{~m} / \mathrm{s}\right.$, several positions). The coalescence mechanism increases the size of the beads and therefore the scale $d_{\text {max }}$. The shift of the $d_{\text {max }}$ pdf when $V_{q}$ decreases reported in Fig. 7 suggests an increase of the number and degree of coalescence events. We believe that the symmetry loss of the pdf in Fig. 7 is an illustration of this point.

The application of the model given by Eq. (6) may help understanding the evolution of $S P$ and identifying the coalescence mechanism. This model represents one unit pattern of the BOAS, i.e., one string plus one bead, the latter being more or less spherical according to the value of the parameter $n$. If the evolution of this unit pattern is only due to the capillary contraction of the string controlled by the viscoelastic forces, a part of the string volume will transfer in the bead but the volume of the unit pattern will remain constant. Thus, the parameter $\alpha(t)$ will decrease according to Eq. (7). However, if a coalescence event occurs, the volume of the unit pattern increases and the parameter $\alpha(t)$ should decrease more rapidly than in the previous case. Furthermore, the parameter $n(t)$ of the model gives an information on the shape of the beads and should indicate whether they are more or less spherical according to the velocity. Thus, the model given by Eq. (6) is applied in order to examine the variation of the parameters $\alpha(t)$ and $n(t)$.

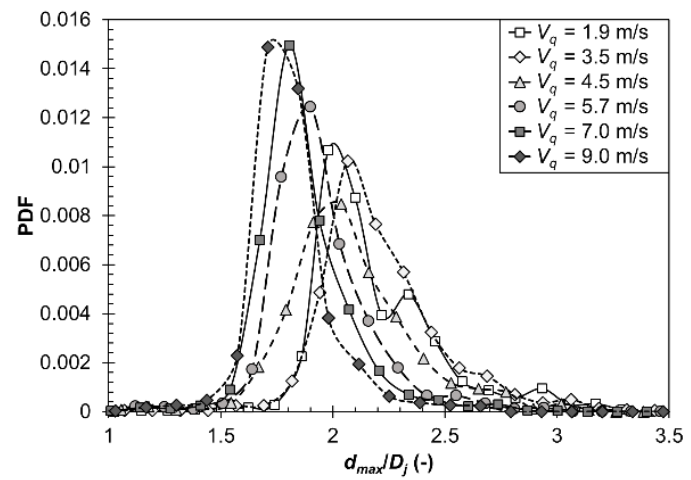

Figure 7. PDF of $d_{\max } / D_{j}$ of the droplets. Influence of the velocity

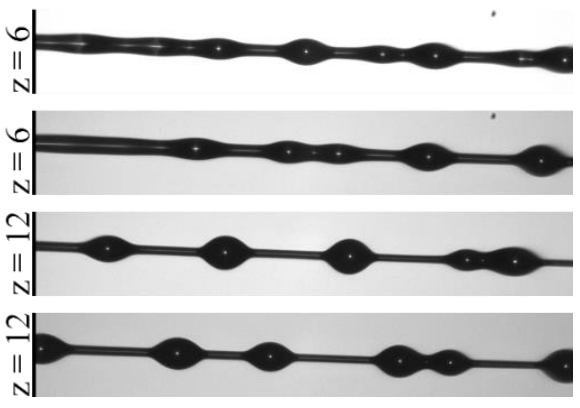

Figure 8. Illustrations of the coalescence mechanism $\left(V_{q}=1.9 \mathrm{~m} / \mathrm{s}\right)$

The application of the model was performed as follows. Equation (6) is used to express $e_{3}(0, t)$ and $e_{3}{ }^{\prime}(0, t)$. (The prime indicates a derivative in the scale space.) It comes:

$$
\left\{\begin{array}{c}
e_{3}(0, t)=\alpha(t) \frac{2}{D_{1}(t)}+(1-\alpha(t)) \frac{n(t)}{D_{2}(t)} \\
e_{3}^{\prime}(0, t)=-\alpha(t) \frac{2}{D_{1}(t)^{2}}-(1-\alpha(t)) \frac{n(t)(n(t)-1)}{D_{2}(t)^{2}}
\end{array}\right.
$$

The quantities $e_{3}(0, t)$ and $e_{3}{ }^{\prime}(0, t)$ are given by the experiments. The parameter $D_{2}(t)$ that represents the maximum scale is taken equal to $d_{\max }(t)$. Finally, the parameter $D_{1}(t)$ is taken equal to the characteristic scale $d_{4}$ that is introduced in Fig. 2. The reason for this choice is that this scale allows us to obtain very good fits. The parameters $\alpha(t)$ and $n(t)$ are found by solving the system Eq. (9). At each time we paid attention that $0 \leq \alpha(t) \leq 1$ and $2 \leq$ $n(t) \leq 3$. The agreement between the model and the experiments is satisfactory as illustrated in Fig. 5 .

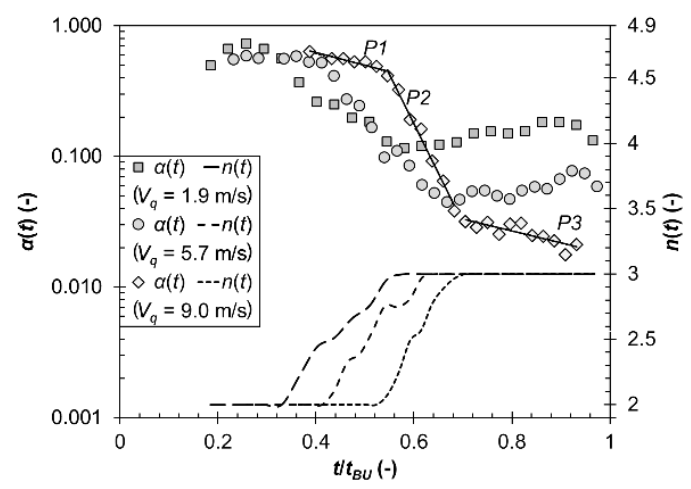


Figure 9. Parameters $\alpha(t)$ and $n(t)$ versus $t$ from the beginning of the elasto-capillary regime to the breakup time. Influence of the velocity. (The symbols correspond to the parameter $\alpha(t)$ and the dashed lines to the parameter $n(t)$ )

Figure 9 presents the parameters $\alpha(t)$ and $n(t)$ for three velocity as a function of the ratio $t / t_{B U}$. For all velocities, the parameter $\alpha(t)$ shows a sharp exponential decrease (P2 in Fig. 9) whose slope depends on the velocity. The characteristic time $t_{2}$ of this decrease $\left(=-t_{B U} /(\right.$ slope P2)) are given in Table 1 for each velocity. These times can be compared with $3 t_{r} / 2$ also given in Table 1 where $t_{r}$ is the characteristic time obtained from the analysis of the scale $d_{1}$ and shown in Fig. 3 (see previous section). For the high velocities (form $7 \mathrm{~m} / \mathrm{s}$ to $10 \mathrm{~m} / \mathrm{s}$ ) we see that $t_{2}<1.5 t_{r}$. This says that the behaviour P2 is the region where the mechanism of coalescence is the more active. This mechanism is fast and produces strings on which the capillary contraction takes place. For the other velocities $(1.9 \mathrm{~m} / \mathrm{s}$ to $5.7 \mathrm{~m} / \mathrm{s}), t_{2}$ is more of the order of $1.5 t_{r}$. This says that the $t_{r}$ measured for these velocities is a characteristic time of the coalescence mechanism and not of the extensional relaxation time as expected. The coalescence is slow and produces strings that are not subject to the elasto-capillary regime of contraction.

The behaviour P2 is preceded by a P1 behaviour (not found for $1.9 \mathrm{~m} / \mathrm{s}$ ). For high velocities, we see in Table 1 that the corresponding characteristic time $t_{1}$ is of the order of $1.5 t_{r}$. The mechanism found here corresponds to the elasto-capillary regime of contraction. However, for low velocities, $t_{1}>>1.5 t_{r}$ : the string is not in the elasto-capillary regime of contraction.

Table 1. Characteristic times of the linear behaviours in Fig. 9 (all velocities)

\begin{tabular}{c|c|c|c|c}
\hline \hline$V_{q}(\mathrm{~m} / \mathrm{s})$ & $t_{r}\left(3 t_{r} / 2\right)(\mathrm{ms})$ & $t_{1}(\mathrm{~ms})$ & $t_{2}(\mathrm{~ms})$ & $t_{3}(\mathrm{~ms})$ \\
\hline \hline 1.9 & $2.18(3.27)$ & - & 2.3 & - \\
3.5 & $1.07(1.61)$ & 7.14 & 0.75 & - \\
4.5 & $0.91(1.37)$ & 3.86 & 0.89 & - \\
5.7 & $0.79(1.19)$ & 5.65 & 0.71 & - \\
7.0 & $0.64(0.96)$ & 0.88 & 0.26 & 29 \\
9.0 & $0.47(0.71)$ & 1.40 & 0.29 & 2.4 \\
10. & $0.43(0.65)$ & 0.96 & 0.16 & 2.4
\end{tabular}

Finally, after the behaviour P2, two situations occur. For the low velocities, $\alpha(t)$ becomes almost constant: the coalescence has stopped and the volume of the ligament is rather constant. Therefore, the continuous thinning of the ligament reported by the scale $d_{1}$ [7] corresponds to an elongation mechanism probably due to gravity effects. For the high velocities, the behaviour P3 is a third exponential decrease whose characteristic time $t_{3}>1.5 t_{r}$ (see Table 1). This mechanism is not the elasto-capillary regime of contraction anymore.

The temporal evolution of $n(\mathrm{t})$ (Fig. 9) shows that it increases from 2 to 3 during the coalescence mechanism. In other words, for every velocity, $n(t)=3$ when the coalescence is over saying that the beads are reasonably spherical. Therefore, the high values of the parameter $S P$ reported in Fig. 6-b for the small velocity $V_{q}$ is due to a non-negligible contribution of the string to this quantity. This is confirmed by the fact that the string at small velocity contain more liquid than at high velocity (see Fig. 9). Images confirm that strings at small velocity are longer than those at high velocity.

\section{Conclusions}

This work completes a previous experimental study of the behaviour of free-falling liquid jet of dilute viscoelastic solutions [1]. This study considered the dynamic at small scales that was detected by applying a 2D multi-scale description of the jets and identified an exponential decreasing regime from which the viscoelastic relaxation time of the solution could be measured. The objective of the present complement is to understand why this relaxation time, which is expected to be a constant physical property of the viscoelastic solution, shows a sharp increase at small jet velocities. To achieve this, the 2D multi-scale description is extended to a 3D multi-scale one. This extension is possible because the capillary instability that develops on a cylindrical liquid jet is an axisymmetric mechanism. This characteristic has been validated here despite a slight initial deformation of the jets mainly observed at high velocity. Among other results, the 3D analysis reports the temporal evolution of the specificsurface-area (interface area per unit volume) of the jet and highlights a deep variation of this evolution when the jet velocity decreases. This evolution is found to be accompanied by an increasing deviation from sphericity of the system. On the other hand, a mechanism of swell-coalescence, whose intensity increases for small velocities, has been identified. The interpretation of these results has been conducted from the application of a simple model of the jet deformation, i.e., of the 3D scale distribution. The model demonstrates that the dynamic of coalescence is very much velocity dependent and that, at low velocity, this mechanism prevents the elasto-capillary contraction at 
small scales from occurring. Furthermore, at low velocity it is found that after the coalescence mechanism, a pure elongation mechanism takes place at small scales that conserves the volume of the strings. In conclusion, the volume of the strings when the breakup is approached is much higher at low velocity explaining the corresponding high values of the specific-surface-area for these cases. These conclusions constitute another demonstration of the practical interest of the multi-scale description and analysis for free liquid flows. The next step for this work is to investigate the coalescence mechanism on free-falling jets of dilute polymer solutions in order to control it when the viscoelastic relaxation time wants to be measured.

\section{Acknowledgments}

The authors acknowledge the financial support from the Frend National Research Agency (ANR) through the program Investissement d'Avenir (ANR-10 LABX-09-01), LABEX EMC3.

\section{References}

[1] Tirel, C., Renoult, M.C., Dumouchel, C., Lisiecki, D., Crumeyrolle, O., Mutabazi, I., (2017) Journal of NonNewtonian Fluid Mechanics (under revision).

[2] Goldin M., Yerushalmi J., Pfeffer R., Shinnar R., (1969) Journal of Fluid Mechanics, 38, pp. 689-711

[3] M. Stelter, G. Brenn, A.L. Yarin, R.P. Singh, F. Durst, (2000) Journal of Rheology, 44, pp. 595-616.

[4] Keshavarz, B., Sharma, V., Houze, E. C., Koerner, M. R., Moore, J. R., Cotts, P. M., Threlfall Holmes, P., McKinley, G. H., (2015) Journal of Non-Newtonian Fluid Mechanics, 222, pp. 171-189.

[5] Christanti, Y., Walker, L.M., (2001) Journal of Non-Newtonian Fluid Mechanics 100(1), pp. 9-26.

[6] Schümmer, P., Tebel, K.H., (1982) Journal of Rheology, 26(1), pp. 77-78.

[7] Schümmer, P., Tebel, K.H., (1982) Rheologica Acta, 21(4-5), pp. 514-516.

[8] Schümmer, P., Tebel, K.H., (1983) Journal of Non-Newtonian Fluid Mechanics, 12(3), pp. 331-347.

[9] Dumouchel, C., Grout, S., (2009) International Journal of Multiphase Flow 35, pp. 952-962.

[10] Dumouchel, C., Aniszewski, W., Ménard, T., Vu, T.T, August 23-27. 2015, $13^{\text {th }}$ ICLASS Conference, Tainan (Taiwan).

[11] Dumouchel, C., Aniszewski, W., Vu, T.T, Ménard, T., (2017) International Journal of Multiphase Flow, 92, pp. 181-192.

[12] Bérubé, J., Jébrak, M., (1999) Comput. Geoscie. 25, pp. 1059-1071.

[13] Dumouchel, C., (2017) ASME - FEDSM, Fluids Engineering Summer Conference, FEDSM2017-69590

[14] Evers, L.W., SAE Technical Series (1994) Paper n`940190. 